\title{
ADAPTACIÓN DE ESTÁNDARES INTERNACIONALES A LAS DELIMITACIONES TERRITORIALES: LÍNEAS LÍMITE JURISDICCIONALES
}

\section{ADAPTATION OF INTERNATIONAL STANDARDS TO TERRITORIALS BOUNDARIS: LOCAL JURISDICCIONALS LINES BOUNDARY}

\author{
Ramon Iborra-Fabra*, Carmen Femenia-Ribera, Gaspar Mora-Navarro \\ Departamento de Ingeniería Cartográfica, Geodesia y Fotogrametría, Universitat Politècnica de València, Camino de Vera s/n, 46022 \\ Valencia.raibfab@doctor.upv.es; cfemenia@cgf.upv.es; joamona@cgf.upv.es
}

\begin{abstract}
:
Disagreements between public administrations and neighbors in areas near the territorial boundaries between municipalities have increased as the urban development of municipalities increases. Not surprisingly, in Spain, the cartographic representation of jurisdictional boundary lines, acting as territorial delimitations between municipalities, were made at the beginning of the 20th century and it is estimated that they are accurate, in some cases, up to 50 meters. These disagreements have been expressed in newspaper publications, in requests for demarcation to administrative bodies, or in court. Focusing on the geographical scope of the Valencian Community, the compilation of publications for the study can promote citizen empowerment and the strengthening of public administrations, in the sense of the Sustainable Development Goals and Targets (SDGs). The adaptation and application of international standards, such as the Land Administration Data Model (LADM) and the International Standard for the Measurement of Land and Land Rights (ILMS), which bring together a framework according to the purpose of the administration (FFP), of the property and of the territory itself, together with public - private partnership (PPP) can be a dynamic fact in the review and improvement of jurisdictional boundary lines.
\end{abstract}

Key words: territorial boundary, municipal boundary. boundarie, ILMS, LADM, PPP, ODS

Resumen:

Las desavenencias entre administraciones públicas y vecinos en las zonas próximas a las delimitaciones territoriales entre municipios han ido en aumento a medida que el desarrollo urbanístico de los municipios ha sido mayor. No en vano, en España, la representación cartográfica de las líneas límite jurisdiccionales, entendidas estas como delimitaciones territoriales entre municipios, fue realizada a principios del siglo XX y se estima que tienen una exactitud, en algunos casos de hasta de 50 metros. Estas desavenencias han sido manifiestas en publicaciones en periódicos, en peticiones de deslinde a los órganos administrativos o en sentencias judiciales. Centrándonos en el ámbito geográfico de la Comunidad Valenciana, la recopilación de las publicaciones para su estudio posterior puede promover la representación ciudadana ciudadana y el fortalecimiento de las administraciones públicas, en el sentido de los objetivos y metas por el desarrollo sostenible (ODS). La adaptación y aplicación de estándares internacionales, como el modelo de datos de la administración del territorio (LADM) y el estándar internacional para la medición de la tierra y de sus derechos (ILMS), que aúnen un marco de trabajo acorde al propósito de la administración (FFP), de la propiedad y del propio territorio, junto con la asociación público - privada (PPP) pueden ser un hecho dinamizador en la revisión y mejora de las líneas límite jurisdiccionales.

Palabras clave: delimitación territorial, línea límite, linde, asociación público - privada (PPP), ILMS, LADM, ODS

\section{Introducción}

En España, las delimitaciones territoriales entre municipios fueron monumentadas a partir de hitos o mojones en los inicios del siglo XX mediante el Decreto del Ministerio de Gobernación de 23 de diciembre de 1870 publicado en la Gaceta de Madrid del 12 de febrero de 1871 (Fig. 1). Posteriormente, se realizaron las acciones de ratificación de los mojones y de las alineaciones que los unen, por las comisiones formadas por los representantes políticos y civiles de cada municipio. Siendo el resultado de estas ratificaciones las «Actas de Deslinde» (Fig. 2).

Trabajos topográficos para la observación de los mojones y su representación en la primera edición del Mapa Topográfico Nacional a escala 1:50.000 (MTN50) se realizaban con posterioridad a la ratificación de estos por los representantes de cada corporación municipal. Cabe indicar que, inicialmente se representaron a escala 1:25.000 junto con las principales vías de comunicación y de elementos hidrográficos en los Planos Geométricos de cada municipio, conocidos como las "Pañoletas», realizados por el Instituto Geográfico y Estadístico. Se

*Corresponding Author: Ramon Iborra-Fabra, raibfab@doctor.upv.es 
observaron y representaron de forma indistinta a si hubo o no ratificación de la delimitación territorial municipal. La exactitud con que se representaron es acorde a la escala de representación. La exactitud con que se representan los mojones en el MTN50 es de hasta 50 metros, así como de las alineaciones que los unen. Mediante las Actas de Deslinde y de los Cuadernos de Campo se trató de cubrir una finalidad administrativa, (Fig 2). Con el paso del tiempo, para las distintas ediciones del MTN50, se empleó el copiado y digitalización de la representación inicial.

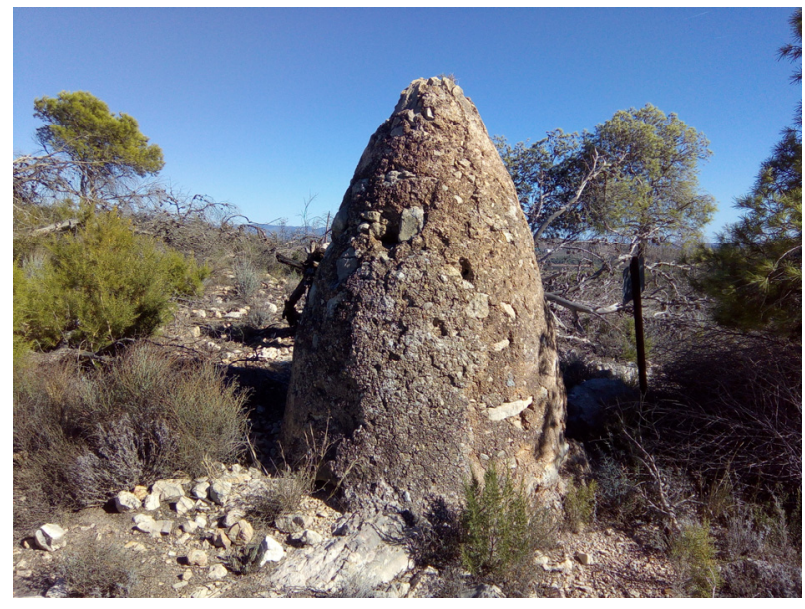

Figura 1: Mojón M3 de la línea límite de Bugarra y Llíria. Fuente: propia.

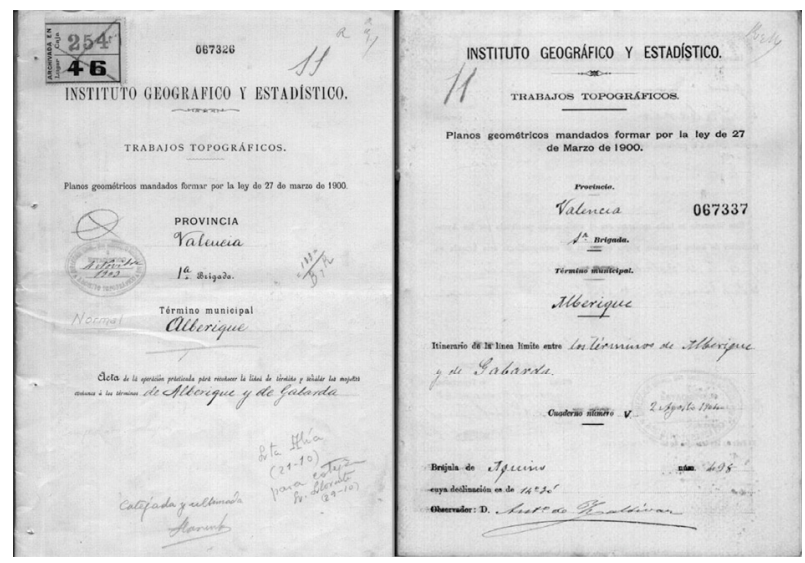

Figura 2: Acta de Deslinde y Cuaderno de Campo de la linea límite de Alberic y Gavarda. Fuente: IGN.

De forma paralela, en la misma época, se empezaron a realizar los trabajos topográficos para la obtención de los Planos Topográficos para el Catastro Parcelario, por polígonos, de los municipios españoles. Estos planos fueron realizados por el Instituto Geográfico y Catastral a escala 1:2.000 y su finalidad fue fiscal pues se trató de representar y catalogar el aprovechamiento que cada ciudadano hacia del territorio. De entre los elementos que se representaron en los Planos citados, se haya la delimitación territorial municipal. Es por ello, que los elementos característicos de las delimitaciones territoriales como son los mojones fueron objeto de trabajos topográficos para finalidades, ya sea fiscal o jurisdiccional, y exactitudes distintas.

En la actualidad, aún se manifiestan las desavenencias entre municipios por revindicaciones del territorio, siendo la delimitación territorial entre ellos el motivo de la disputa. Disputas que se ven incrementadas por la planificación urbanística del territorio. La indeterminación de las delimitaciones territoriales municipales ya sea por la desatención de la conservación de los mojones, por errores u omisión en su representación o por la falta de acuerdo entre los municipios; no ayuda a solucionar el conflicto.

Por ello, la recopilación de las publicaciones de noticias, de peticiones de deslinde a los órganos administrativos o de sentencias judiciales para su estudio posterior puede promover la representación y el fortalecimiento de las administraciones públicas, en el sentido de los objetivos y metas por el desarrollo sostenible (ODS).

Un fortalecimiento que puede verse incrementado por la adaptación y aplicación de estándares internacionales, como el modelo de datos de la administración del territorio (LADM) y el estándar internacional para la medición de la tierra y de sus derechos (ILMS), que aúnen un marco de trabajo acorde al propósito de la administración (FFP), de la propiedad y del propio territorio, junto con la asociación público - privada (PPP) pueden ser un hecho dinamizador en la revisión y mejora de las líneas límite jurisdiccionales.

Mediante las asociaciones público - privadas se fomenta el emprendurismo profesional y la participación ciudadana en la Administración del Territorio, así como la optimización de los recursos económicos y la conclusión de proyectos en periodos concretos de tiempo. Para ello, es necesaria la homogenización de la información evaluada y generada que permita el control, revisión y posterior tratamiento de los datos. Por ello, la estandarización de procesos y de la información puede ayudar en la realización de proyectos complejos.

Siendo la Comunidad Valenciana el ámbito geográfico de aplicación de los estudios, el objetivo de este artículo es describir la recopilación documental de los convenios de la participación público - privada para la administración del territorio en España, de los estándades internacionales que son de aplicación, así como los procedimientos administrativos mediante los cuales se realizan los trabajos de recuperación y mejora geométrica de las líneas límite en la actualidad.

\section{La Asociación Público-Privada en la Administración del Territorio en España}

La Participación o Asociación Público - Privada (PPP), en inglés Public - Private Partnerships, según el Banco Mundial (BM) es «un contrato a largo plazo entre una empresa privada y un organismo para proveer un bien o un servicio público, en el cual la parte privada corre con los riesgos y responsabilidad de la gestión, y la remuneración está vinculada al rendimiento» (The World Bank Group 2021; World Bank 2014).

La aplicación de las PPP en la gestión del territorio ha sido evaluada en la Sesión $8^{a}$ de la Reunión Anual de la Comisión 7 de la International Federation of Surveyors (FIG) celebrada en octubre de 2020 (FIG 2021). La FIG en colaboración con el Working Party on Land Administration (WPLA) revisan los principios generales para el empleo de la PPP como mecanismo para mejorar 
los servicios y la infraestructura de los organismos catastrales y registrales.

En este sentido, en España, cabe destacar el convenio de colaboración entre la Dirección General de Catastro (DGT) y el Colegio Oficial de Ingenieros Técnicos en Topografía (COIGT) mediante la Resolución de 7 de febrero de 2019 el cual se enmarca en temas de la administración de la tierra (Velasco et al. 2021). Este convenio, es fruto de la aplicación del art. 4, del Texto Refundido de la Ley del Catastro Inmobilario y el objeto del convenio es el intercambio de información gráfica y alfanumérica. Tiene una vigencia y renovación anual, con una Comisión de Seguimiento, Vigilancia y Control, así como planes de formación para los colegiados.

Con el Colegio de Registradores de la Propiedad y Mercantiles de España (CORPME), el COIGT tiene un convenio de colaboración desde el 9 de octubre de 2018.

El convenio con el CORPME presenta características distintas al de la DGC pues el objeto es lograr la interoperbilidad entre las plataformas del CORPME y del COIGT pues tiene una vigencia de cuatro años, planes de formación y se realiza al amparo de profesionales acreditados y del protocolo de visados según la Norma ISO 9001:2.015.

Los profesionales acreditados que, en España, en el contexto de la Administración del Territorio, el COIGT ha creado el Esquema de Certificación de Personas, basado en la Norma ISO 17.024 (ISO 2012), con perfil de Técnico Especialista en Catastro, Propiedad Inmobiliaria y Valoración.

El organismo de certificación, acreditado por la Agencia Española de Acreditación (ENAC), que realiza las pruebas es el Instituto de Graduados en Ingeniería e Ingenieros Técnicos de España (INGITE).

\section{Estándares Internacionales, aplicados a la administración del territorio}

Las delimitaciones territoriales españolas, en general, y las líneas límite jurisdiccionales, en particular, forman parte de equipamiento geográfico de referencia español, regulado por la ley 14/2010, de 5 de julio, sobre las infraestructuras de datos y los servicios de información geográfica de España.

La legislación citada es la transposición al estado español de la legislación europea INSPIRE con el fin de definir a infraestructura de datos europea.

En España, es el Instituto Geográfico Nacional (IGN) el órgano que ha adaptado la casuística de las distintas Unidades Administrativas en España al modelo de datos propuesto por la Directiva INSPIRE.

Cabe destacar que, en mayor medida, los municipios están formados por un único territorio, pero también los hay con varios territorios e incluso territorios con jurisdicción compartida. Este hecho es relevante pues plantea el empleo de modelos de datos de administración del territorio (LADM) basados en la Norma UNE-EN ISO 19.152 (UNE 2021).

Sin embargo, en España, dado que ya existe un modelo de datos consolidado, la aplicación de la LADM no se está planteando con relación a grandes cambios estructurales.
La correcta estandarización de las fuentes de datos espaciales y su representación, geométrica y topológica; permitirá proporcionar una base extensible para el desarrollo de los sistemas de la administración de territorio, así como de permitir la comunicación entre los interesados involucrados.

Por otro lado, el desarrollo y adaptación de la norma International Land Measurement Standard (ILMS) (Coalición de las ILMS 2019) a cada país, desarrollado en su primera edición en mayo de 2019, puede ayudar a normalizar la obtención de datos espaciales y los Sistemas de Información Territorial (SIT).

Esta temática ha sido evaluada en la Sesión 9 de la Reunión Anual de la Comisión 7 de la International Federation of Surveyors (FIG 2021), la cual trata de la Administración del Territorio, de los Objetivos de Desarrollo Sostenible (ODS) de las Naciones Unidas, de los modelos de datos de la ISO 19.125 y del Modelo en el Dominio de la Tenencia Social (STDM), así como de las iniciativas con el enfoque adecuado al propósito, fitfor-purpose.

La formación específica de los profesionales acreditados en la Norma ISO 17.024, basada en esquemas de certificación de las personas con competencias profesionales concisas en materia técnico - jurídica es un aliciente para el éxito de la aplicación de los estándares internacionales relacionados con la Administración del Territorio aplicado a las delimitaciones territoriales.

\section{La Recuperación y Mejora Geométrica de las Líneas Límite Jurisdiccionales, en la actualidad}

En 2012, el IGN promovió en las entidades municipales la recuperación y mejora geométricas de las líneas límite. Entendida la recuperación, en aquellos casos en que los mojones o el trazado de esta por vías de comunicación o cursos fluviales había desaparecido; y la mejora geométrica, por la determinación de su posición por técnicas e instrumentación topográfica de mayor precisión que con las que fueron observadas a principios del siglo $X X$.

El objetivo no era otro que incentivar la recuperación y mejora geométrica de las líneas límite. Fruto de estas actuaciones es el procedimiento y pliego de condiciones técnicas para la recuperación y mejora geométrica de las líneas límite jurisdiccionales (Femenia-Ribera et al. 2014).

En el procedimiento y pliego descrito normalizó los distintos procedimientos que debian de seguir las entidades locales para la ratificación del Acta Adicional al Acta de Deslinde original de inicios del siglo XX.

De la misma manera, se establecieron los requisitos técnicos y jurídicos necesarios en caso de que las entidades locales requiriesen la participación público privada de un técnico especialista en la materia (IborraFabra et al. 2020) en cumplimiento de lo dispuesto en el Real Decreto 1690/1986, de 11 de junio, por el que se aprueba el Reglamento de Población y Demarcación de las Entidades Locales.

En la actualidad, el IGN está realizando los trabajos técnicos y asesorando en el referendo jurídico en los 
convenios de colaboración suscritos en las Comunidades Autónomas de Cantabría y de Castilla la Mancha.

\subsection{En la Comunidad Valenciana}

El Instituto Cartográfico Valenciano (ICV), creado en 1997, es un organismo autónomo de la Generalitat y le corresponde, Ley $2 / 2020$, de 2 de diciembre, de la Generalitat, de la información geográfica y del Insttuto Cartográfco Valenciano; el ejercicio de las actividades geomáticas que sean necesarias para el ejercicio de cualquier competencia de la Generalitat.

En el año 2001, se creó el Consejo Técnico de Delimitación Territorial de la Comunitat Valenciana, mediante el Decreto 113/2001, de 19 de junio, siendo posible la participación expertos para el asesoramiento del consejo técnico.

En años recientes, el ICV ha fomentado la contratación pública de los trabajos técnicos para la recuperación y mejora de las líneas límite mediante licitaciones (Institut Cartogràfic Valencià 2016) (Fig. 3). La contratación pública queda regulada por la Ley de Contratos del Sector Público y las empresas licitadoras tienen que estar inscritas en el Registro Oficial de Contratistas y Empresas Clasificadas de la Comunidad Valenciana (ROLECE). Mediante este registro administrativo a los licitadores se les evalúa su capacidad técnica y económico - financiera para realizar la contratación.

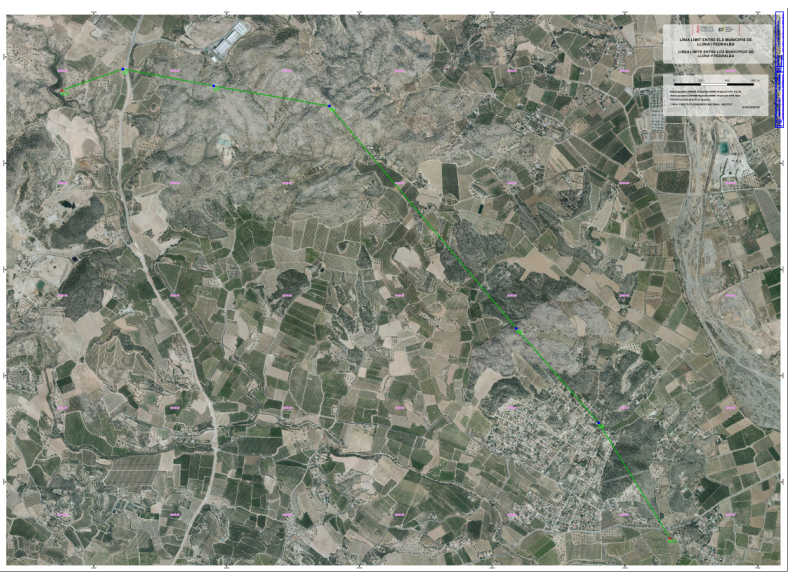

Figura 3: Mejora geométrica de la línea límite de Pedralba y Llíria. Fuente: propia en colaboración con el ICV.

Cabe destacar, que el ICV insta a que los licitadores tengan clasificación CPV, Common Procurement Vocabulary, basada en: 71351810 - Servicios Topográficos, 71353000 - Servicios de mediciones topográficas de superficie y 71355200 - Servicio oficial de topografía catastral.

Con la aprobación de la Ley 2/2020, de 2 de diciembre, de la Generalitat, de la Información Geográfica y del Institut Cartogràfic Valencià, se aprueban los «actos de oficio» a la mejora geométrica de las líneas de término, dado que no supone ni una nueva delimitación ni una alteración, puesto que refleja fielmente el acta de delimitación original aprobada en su día por los ayuntamientos implicados.

Se crea también, mediante la nueva legislación, el Registro de Cartografía autonómico.

\section{Conclusiones}

Con el desarrollo de este estudio se ha desgranado singularidad del estado actual de las línias límite jurisdiccionales. En el sistema cartográfico actual, conviven procedimientos de observación y de referendos jurídicos con distintas finalidades y precisiones técnicas sobre una misma realidad. Es por ello es determinante, la adaptación a las líneas límite jurisdiccionales de los estándares internacionales y en la implementación procedimientos internacionales de medición de la tierra.

La armonización y normalización de los procedimientos de obtención de los datos espaciales, así como de su interacción con los operadores jurídicos, administrativos y la propia ciudadanía permitirá homogenizar los resultados, así como lograr una eficiencia administrativa y económica óptima. Para ello, la participación de público - privada de técnicos especialistas acreditados en normas internacionales y con competencias profesionales en el ámbito de las delimitaciones territoriales, en general, y en las líneas límite municipales, en particular, es significativo. Participación que ha de permitir su aplicación en espacio concreto de tiempo y que cubra la totalidad del territorio.

Queda por abordar, la posibilidad de colaboración institucional con el IGN y el ICV, en este caso, entre organismos públicos para el caso concreto que nos atañe. El estudio de aquellos requisitos técnicos y jurídicos, formales y económico-financieros que sean acordes al vigente sistema de contratación público, así como de la formación, evaluación y seguimiento de estos.

\section{Agradecimientos}

Agradecemos la confianza mostrada en estos estudios a los técnicos y responsables del Instituto Geográfico Nacional y del Instituto Cartográfico Valenciano.

\section{References}

BENNetT, R., MASLI, E., POTEL, J., UNGER, E. M., LEMMEN, C. H. J., and DE ZEEUW, K., 2019. Cadastral Entrepreneurs Recognizing the Innovators of Sustainable Land Administration. In FIG Working Week 2019: Geospatial Information for a Smarter Life and Environmental Resilience.

COALICIÓN DE LAS ILMS. 2019. Coalición de Normas Internacionales de Levantamientos de Tierra. Available: https://ilmscoalition.files.wordpress.com/2020/08/ilms-spanish-final-web-upload.pdf [7/15, 2021].

FEMENIA-RIBERA, C., BENITEZ-AGUADO, E., MORA-NAVARRO, G., and MARTINEZ-LLARIO, J, 2014. Method os recovering munipal boundary lines in Province of Valencia (Spain) by means of histotical cadastral maps. Survey Review, 46(337), pp. 255-266. DOI: 10.1179/1752270613Y.0000000081

FEMENIA-RIBERA, C., MORA-NAVARRO, G., and MARTINEZ-LLARIO, J. C., 2021. Advances in the Coordination between the Cadastre and Land Registry. Land, 10(1), 81. DOI: 10.3390/land10010081 
FIG. 2021. International Federation of Surveyors, IG Commission 7 - activities - events. Available: https://www.fig.net/organisation/comm/7/activities/events/2020_annual_meeting.asp [7/15, 2021].

IBORRA-FABRA, R., FEMENIA-RIBERA, C., and MORA-NAVARRO, G., 2020. La participación público-privada en la recuperación y mejora geométrica de líneas límite municipales. Caso práctico de Alberic y Gavarda, Valencia (España). Mapping, 201, pp. 16-21.

INSTITUT CARTOGRÀFIC VALENCIÀ. 2016. Delimitació municipal - Generalitat Valenciana (gva.es). Available: https://icv.gva.es/va/delimitacio-municipal [7/15, 2021].

ISO. 2012. ISO/IEC 17024:2012(es), Available: https://www.iso.org/obp/ui\#iso:std:iso-iec:17024:ed-2:v1:es [7/15, 2021].

MAHONEY, R., MOSS, D., JACUBEIT, T., ARONSOHN, A., GONZALEZ, A., TIWARI, P., and SMITH, A., 2019. International Land Measurement Standard: Due Diligence for Land and Real Property Surveying.

THE WORLD BANK GROUP. 2021. Home | PPP Knowledge Lab. Available: https://pppknowledgelab.org [7/15, 2021].

UNE. 2021. Información geográfica. Modelo para el ámbito de la administración del territorio (LADM). (ISO 19152:2012).

VELASCO MARTÍN-VARES, A., FEMENIA-RIBERA, C., GARCÍA BENITO, J., and OJEDA MANRIQUE, J., 2021. Finally, the model of collaboration between land surveyor engineer and the Spanish Directorate General for Cadastre is institutionalized. Commission 7 FIG 2021

WORLD BANK DOCUMENT. 2014. Public-Private Partnerships. Reference Guide, Version 2.0. Available: http://documents1.worldbank.org/curated/en/600511468336720455/pdf/903840PPP0Refe0Box385311B000PUBLIC0 .pdf $[7 / 15,2021]$. 